\title{
Diagnóstico Del Aprendizaje Electrónico Móvil (Podcast) Para La Comprensión Auditiva Del Inglés
}

\author{
Edison Renato Ruiz López \\ Patricia Pilar Moyota Amaguaya \\ Magíster en Lingüística y didáctica de la enseñanza de Idiomas Extranjeros \\ Docente del Centro de Idiomas \\ de la Escuela Superior Politécnica de Chimborazo
}

\section{Doi: 10.19044/esj.2018.v14n1p134 URL:http://dx.doi.org/10.19044/esj.2018.v14n1p134}

\begin{abstract}
The objective of this research was to evaluate the listening comprehension of the English language through the Mobile electronic Learning (Podcast). A diagnosis was applied to teachers who teach the English language in the Pedro Vicente Maldonado Educational unit of the city of Riobamba. To this end, we analyzed the methodological principles that guide the use of podcast as a didactic tool in the teaching of the English language with emphasis on the dexterity of listening. For the podcast application, multimedia resources were used on mobile devices; while the evaluation was carried out over an eight-week period. The research is descriptive, so the information was developed in a precise and systematic way. The collection of information was through a survey aimed at teachers, the same one that underwent review and was endorsed by academic peers; the analysis of the data was through descriptive statistics, that is to say that the obtained information was tabulated, interpreted and represented by means statistical tables and graphs. Finally, it was determined that the podcast is useful in listening comprehension in the English language learning process, as it constitutes an entertaining and multifunctional technique for teaching..
\end{abstract}

Keywords: Podcast, listening comprehension, English

\section{Resumen}

El objetivo de la presente investigación, fue evaluar la comprensión auditiva del idioma inglés a través del aprendizaje electrónico móvil (Podcast). Se aplicó un diagnóstico dirigido a docentes que enseñan el idioma inglés en la Unidad Educativa Pedro Vicente Maldonado de la ciudad de Riobamba. Para ello, se analizó los principios metodológicos que guían el uso de podcast como herramienta didáctica en la enseñanza del idioma inglés con énfasis en 
la destreza del listening. Para la aplicación del podcast, se utilizó recursos multimedia en dispositivo móviles; mientras que la evaluación se realizó durante un periodo de ocho semanas. La investigación es descriptiva, por lo que la información fue desarrollada de forma precisa y sistemática. La recolección de la información, fue mediante una encuesta dirigida a los docentes, la misma que se sometió a revisión y fue avalada por pares académicos; $\mathrm{El}$ análisis de los datos fue a través de estadística descriptiva, es decir que la información obtenida fue tabulada, interpretada y representada mediante tablas y gráficos estadísticos. Finalmente se determinó, que el podcast es útil en la comprensión auditiva dentro del proceso de enseñanza aprendizaje del idioma inglés, pues constituye una técnica entretenida y multifuncional para la enseñanza.

Palabras-Claves: Podcast, comprensión auditiva, Inglés

\section{Introducción}

Debido a la constante aplicación de métodos retrógradas y aburridos que salen absolutamente del contexto actual del mundo real, el internet ha permitido durante los últimos años, experimentar y aplicar un sinnúmero de oportunidades de aprendizaje para los estudiantes, sobre todo para quienes han encontrado en el aprendizaje del idioma inglés una real problemática,

Partiendo de la dificultad que tienen los estudiantes al momento de escuchar una pregunta simple, un diálogo, o una conversación en inglés, es decir, en comprender el idioma; la presente investigación pretende determinar cómo la utilización del Aprendizaje Electrónico Móvil (Podcast) puede facilitar la comprensión del inglés. Por lo que las herramientas tecnológicas y nuevas tecnologías de la información (TIC) son aplicadas en el desarrollo de la compresión auditiva y son utilizadas por los docentes de la Unidad Educativa Pedro Vicente Maldonado para la enseñanza del idioma inglés. De ahí la importancia del Podcast como recurso adicional en la enseñanza del inglés, para que los docentes del área de lengua extranjera puedan reforzar los conocimientos de los estudiantes del Segundo Año de Bachillerato de la Unidad Educativa Pedro Vicente Maldonado, y desarrollar su destreza auditiva.

El Aprendizaje Electrónico Móvil (Podcast) al ser una herramienta auditiva, permite ayudar a desarrollar las destrezas del idioma inglés, a través de interesantes opciones tales como: cuentos, noticias, canciones, etc., las mismas que ayudan a los estudiantes a mantenerse interesados en la temática propuesta por el docente en el contenido de dicha herramienta.

The University of Austin at Texas (2013) propuso una definición de cuatro partes: "un Podcast es un audio digital o un archivo de video que es episódico, descargable, que corre en algún programa, principalmente con un 
conductor o tema, y normalmente vía un alimentador automático con un software computacional".

"La palabra Podcast proviene de la mezcla en inglés de iPod (gadget con el que se hicieron populares) y Broadcast (difundir contenidos)" María del Mar Sánchez Vera. Universidad de Murcia, ed. «Objetos de Aprendizaje de la asignatura Diseño y Evaluación de Materiales Educativos». Consultado el 21 de abril de 2015.

La tesis doctoral presentada por Francisco Martín Álvarez, de la Universidad Nacional de Educación a Distancia de Madrid, en el año 2014, misma que tituló: "El podcasting en la enseñanza de las lenguas extranjeras", arrojó como resultado que el uso de Podcast en el área de lenguas extranjeras es más beneficioso que el sistema tradicional. Numerosos estudios confirman su eficacia en la enseñanza de lenguas a través de las nuevas tecnologías y reclaman un lugar para la integración del podcasting, que aumenta el rendimiento y fomenta la motivación en el aprendizaje de la lengua en general, incluidos los aspectos de naturaleza cultural o histórica.

Aunque en un principio era necesario tener uno de estos aparatos para escucharlos, hoy ya no es necesario y basta con instalar cualquier reproductor de audio en cualquier sistema operativo. De hecho, la mayoría de los Podcast se pueden escuchar directamente desde la web.

El uso didáctico de los podcast enriquece la experiencia de aprendizaje (Boulos, Maramba y Wheeler, 2006), potencia la atención del estudiante y su reflexión (Baird y Fisher, 2006), incrementa su satisfacción (Miller y Piller, 2005) e, inclusive, reduce la ansiedad (Chan y Lee, 2005).

Estos programas en episodios comenzaron a hacerse popular con la masificación del iPod; sin embargo, en febrero del 2004 el periódico inglés The Guardian ya hablaba de este tipo de nuevo medio. Adam Curry, VJ estadounidense de MTV, es quien se cree ideó este tipo de plataforma y años después fundó su empresa Mevio, plataforma web que agrupa cientos de podcast y videocasts.

El Podcast no es un archivo u objeto de aprendizaje único, pues se trata de una actualización continua de archivos. Es importante no confundir Podcast con episodio; la diferencia entre ambos estiba en que Podcast es "un programa, compuesto por sucesivas ediciones, que se pueden sindicar para tener que bajar aquellas que ya se han oído" (Marcelo y Martín, 2008: 32) y un episodio "es el nombre de cada una de las ediciones del podcast. La diferencia sería igual a la existente entre una serie de televisión y cada uno de sus capítulos" (Marcelo y Martín, 2008: 32).

El uso didáctico del podcast enriquece la experiencia de aprendizaje (Boulos, Maramba y Wheeler, 2006), potencia la atención del estudiante y su reflexión (Baird y Fisher, 2006), incrementa su satisfacción (Miller y P iller, 2005) e, inclusive, reduce la ansiedad (Chan y Lee, 2005). 
Además, la utilización del podcasting con finalidades didácticas presenta una serie de potencialidades asociadas al enriquecimiento del propio proceso de enseñanza aprendizaje, así como a una nueva concepción de éste.

En el e-Learning, "el término distancia implica un cambio geográfico entre donde residen los contenidos y el lugar en el que se toman, manteniendo siempre una conexión física entre ellos. En cambio, en el m-Learning el término distancia implica que la recuperación o el acceso al contenido puede hacerse en movimiento, sin importar el lugar y obteniendo un mayor provecho del tiempo disponible". Rocael Hernández y Miguel Morales (2009). Universidad de Galileo. Artículo "Dispositivos móviles en la Educación"

Las facilidades de acceso y consumo que ofrecen los podcasts, particularmente desde la perspectiva de los dispositivos móviles, permiten crear oportunidades para la revisión de los contenidos académicos, impensables en un contexto educativo convencional. Su utilización favorece la flexibilización de la enseñanza que sobrepasa los límites del aula y potencia un acceso diacrónico, a medida del estudiante. Un usuario que muestra una predisposición positiva hacia los contenidos educativos vehiculados en formato podcast y que debe ser aprovechada por los docentes para entrar en sintonía con las nuevas generaciones de discentes, los llamados nativos digitales (Moura y Carvalho, 2006, p. 2).

Cuando se habla de la Web 2.0, "nos referimos a una serie de aplicaciones y páginas de Internet que utilizan la inteligencia del usuario para proporcionar servicios interactivos en red dando a éste el control de sus datos" (CDI, 2015). La primera etapa del Internet facilitaba el acceso a una gran cantidad de información pero existía en su uso una limitada capacidad del usuario para enviarla, manejarla o transformarla.

Por este motivo, dentro de la presente investigación se considera importante la evaluación y el análisis de la comprensión auditiva del idioma inglés, a través de la utilización del Podcast, como herramienta tecnológica de aprendizaje, lo cual concuerda con el modelo educativo futurista y globalizado, aplicado en el mundo entero.

\section{Metodología}

La presente investigación es socioeducativa debido a se orienta hacia la producción de conocimiento (educación) y se aplicó con los docentes de la Unidad Educativa Pedro Vicente Maldonado de la ciudad de Riobamba.

El proceso investigativo está plasmado en la evaluación del campo de conocimientos, a fin de conocer el campo de estudio. Se analizó los factores que condicionan la comprensión auditiva del idioma inglés a través del podcast como principio metodológico que guían el uso de podcast como herramienta didáctica. 
La investigación se enmarca en el enfoque cuali-cuantitativo. Es cualitativo debido a que se realizó un análisis bibliográfico, lo cual permitió obtener referencias investigativas acerca del Aprendizaje Electrónico Móvil (Podcast), realizadas a nivel local e internacional. Este proceso de recolección de información fue útil para la construcción del objeto de la investigación; la investigación bibliográfica y documental ocupa un lugar importante, ya que garantiza la calidad de los fundamentos teóricos de la investigación.

El estudio conjuga el análisis interpretativo acorde a resultados obtenidos en base a una encuesta con respuestas arrojadas a una encuesta, aplicada a la población investigada. Para ello, se recurrió a estadística descriptiva, a fin de comprobar los objetivos planteados en cuanto al uso de del Podcast y la comprensión auditiva del inglés.

La selección de los sujetos de estudio no es al azar pues está dirigida a los docentes de la Unidad Educativa Pedro Vicente Maldonado durante el año lectivo 2016-2017.La investigación recabó información de los docentes, para analizar la comprensión auditiva del idioma inglés posterior al uso del Podcast como herramienta de refuerzo en el proceso de enseñanza aprendizaje. Para esto se utilizaron métodos cualitativos (encuesta) y el respectivo análisis bibliográfico; que interactuaron con los datos cuantitativos (resultados estadísticos de los test aplicados) y se apoyaron mutuamente en la dilucidación del problema.

Para realizar la investigación se realizó un acercamiento con las autoridades de la unidad educativa para explicar su importancia. Posteriormente se aplicaron las actividades mediante el uso de lo podcast para la comprensión auditiva en el idioma inglés en un periodo de 8 semanas para finalmente aplicar la encuesta. Con los datos obtenidos de la encuesta se realizó la tabulación, análisis e interpretación de resultados acompañado de la representación a través de tablas y gráficos estadísticos.

\section{Resultados}

Análisis e interpretación de resultados de la evaluación a los docentes de la Unidad Educativa Pedro Vicente Maldonado de la ciudad de Riobamba.

Ítem 1: ¿Utiliza usted recursos multimedia (video, audio) en dispositivos móviles (teléfonos inteligentes, tablets, computador portátil, iPads) para desarrollar la comprensión auditiva del idioma inglés? 
Tabla 1: Uso de recursos multimedia en dispositivos móviles para desarrollar comprensión auditiva

\begin{tabular}{ccc}
\hline Escalas & Frecuencias & Porcentaje \\
\hline Siempre & 0 & $0 \%$ \\
Casi siempre & 3 & $25 \%$ \\
A veces & 5 & $41 \%$ \\
Rara vez & 2 & $17 \%$ \\
Nunca & 2 & $17 \%$ \\
TOTAL & 12 & $100 \%$ \\
\hline
\end{tabular}

Fuente: Encuesta

Elaborado por: Ruiz Renato, 2017

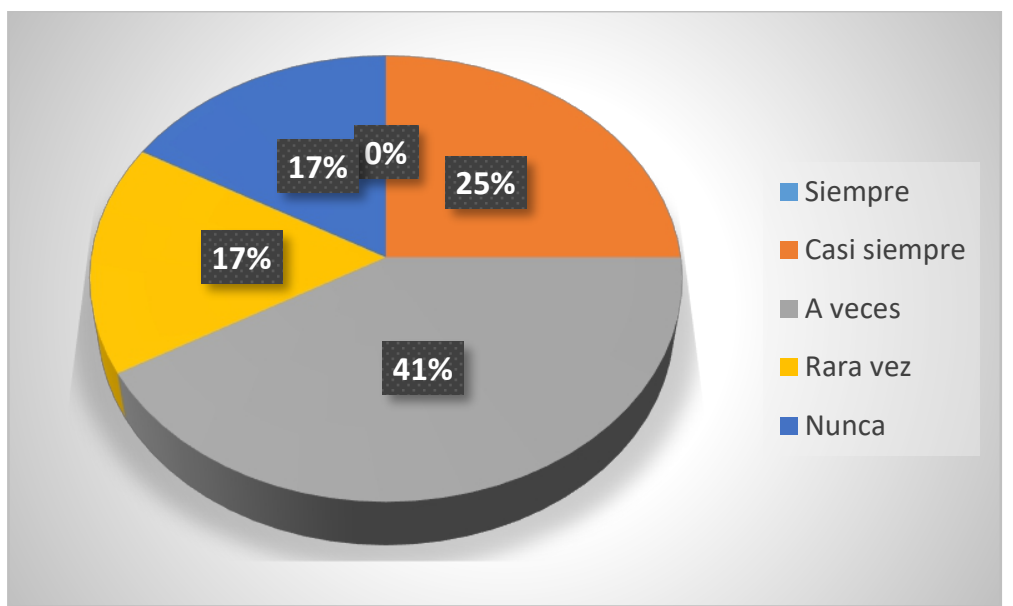

Gráfico 1: Uso de recursos multimedia en dispositivos móviles para desarrollar comprensión auditiva

\section{Análisis e interpretación}

En el ítem 1, el $41 \%$ de los docentes registró que $A$ veces utiliza recursos multimedia (video, audio) en dispositivos móviles (teléfonos inteligentes, tablets, computador portátil, iPads) para desarrollar la comprensión auditiva del idioma inglés, el 25\% Casi siempre, el 17\% Nunca y Rara vez; mientras que no se registró frecuencias en la opción Siempre.

Se puedo observar que la mayoría de ellos utiliza recursos multimedia (A veces) para desarrollar la comprensión auditiva del idioma inglés, y el 25\% casi siempre; es decir, que con los estudiantes con quienes trabajaron, están habituados al uso de recursos multimedia tales como: audio y video, dentro del aula de clase; o al menos conocen de su funcionamiento y/o utilidad.

Este resultado representó una ventaja dentro de la presente investigación, debido que el aprendizaje electrónico móvil (Podcast) utiliza recursos de audio para desarrollar la destreza del lenguaje (listening), y es conveniente que los docentes hayan utilizado estos recursos con cierta frecuencia.

Ítem 2: ¿Utiliza alguna aplicación móvil como herramienta didáctica para reforzar el nivel de comprensión auditiva del idioma inglés? 
Tabla 2. Uso de alguna aplicación móvil como herramienta didáctica para reforzar el nivel de comprensión auditiva del idioma inglés

\begin{tabular}{ccc}
\hline Escalas & Frecuencias & Porcentaje \\
\hline Siempre & 0 & $0 \%$ \\
Casi siempre & 1 & $8 \%$ \\
A veces & 2 & $17 \%$ \\
Rara vez & 6 & $50 \%$ \\
Nunca & 3 & $25 \%$ \\
TOTAL & 12 & $100 \%$ \\
\hline
\end{tabular}

Fuente. Encuesta

Elaborado por. Ruiz Renato, 2017

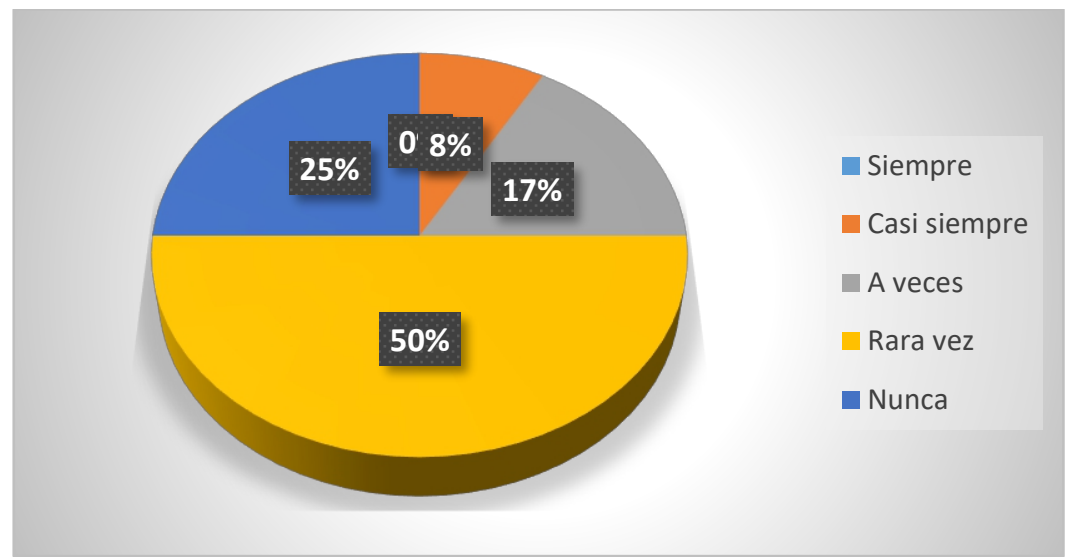

Gráfico 2. Uso de alguna aplicación móvil como herramienta didáctica para reforzar el nivel de comprensión auditiva del idioma inglés

\section{Análisis e interpretación}

En el ítem 2, el 50\% de los decentes registró que Rara vez utiliza alguna aplicación móvil como herramienta didáctica para reforzar el nivel de comprensión auditiva del idioma inglés, el 25\% Nunca, el 17\% A veces; el $8 \%$ Casi siempre; mientras que no se registró frecuencias en la opción Siempre.

Es visible que la mitad de docentes del Área de Lengua Extranjera rara vez ha utilizado alguna aplicación móvil como herramienta didáctica para reforzar el nivel de compresión auditiva del idioma inglés; mientras que 3 docentes nunca han utilizado alguna aplicación móvil como refuerzo al nivel de la comprensión auditiva. Esto quiere decir que, el tipo de aprendizaje electrónico móvil (Podcast) que se desea aplicar es muy poco utilizado por los docentes, por lo tanto, también es poco utilizado por los estudiantes; convirtiéndose en una herramienta novedosa y que podría llamar la atención dentro del aula.

Ítem 3: ¿Cree usted que sus estudiantes comprenden el contenido del material auditivo en clase de inglés? 
Tabla 3. Comprensión de los estudiantes con el contenido del material auditivo en clase de

\begin{tabular}{ccc} 
& inglés \\
\hline Escalas & Frecuencias & Porcentaje \\
\hline Siempre & 0 & $0 \%$ \\
Casi siempre & 2 & $17 \%$ \\
A veces & 3 & $25 \%$ \\
Rara vez & 5 & $41 \%$ \\
Nunca & 2 & $17 \%$ \\
TOTAL & 12 & $100 \%$ \\
\hline
\end{tabular}

Fuente. Encuesta

Elaborado por. Ruiz Renato, 2017

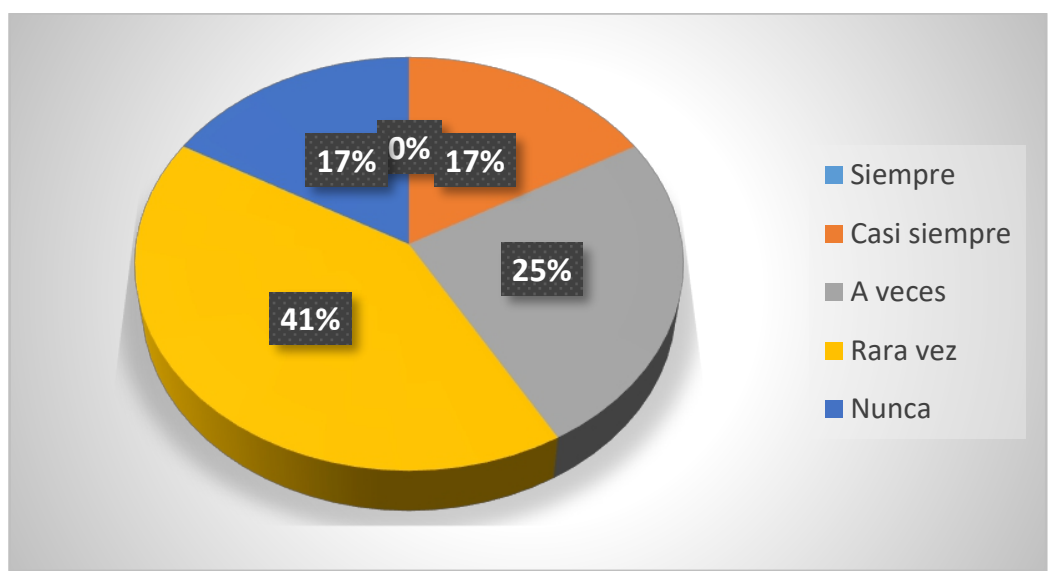

Gráfico 3. Comprensión de los estudiantes con el contenido del material auditivo en clase

\section{Análisis e interpretación}

En el ítem 3, el $41 \%$ de los docentes registró que Rara vez sus estudiantes comprenden el contenido del material auditivo en clase de inglés, el $25 \%$ A veces, el $17 \%$ Nunca; el 17\% Casi siempre; mientras que no se registró frecuencias en la opción Siempre.

Se pudo apreciar que casi la mitad de los docentes, creen que sus estudiantes rara vez comprenden el contenido del material auditivo en clase de inglés; mientras que el $25 \%$ a veces; por lo que en este caso, el aprendizaje electrónico móvil (Podcast) resultaría muy útil para el desarrollo de la compresión auditiva de los estudiantes, debido a sus utilidades y beneficios dentro del proceso de enseñanza aprendizaje de las destrezas del idioma inglés, específicamente de la destreza de la escucha.

Ítem 4: ¿La institución donde usted labora presta servicio de conexión inalámbrica a internet en todos los espacios físicos, para el aprendizaje del inglés? 
Tabla 4. Conexión inalámbrica a internet en todos los espacios físicos, para el aprendizaje del inglés

\begin{tabular}{ccc}
\hline Escalas & Frecuencias & Porcentaje \\
\hline Siempre & 2 & $17 \%$ \\
Casi siempre & 8 & $67 \%$ \\
A veces & 1 & $8 \%$ \\
Rara vez & 1 & $8 \%$ \\
Nunca & 0 & $0 \%$ \\
TOTAL & 12 & $100 \%$ \\
\hline
\end{tabular}

Fuente. Encuesta

Elaborado por. Ruiz Renato, 2017

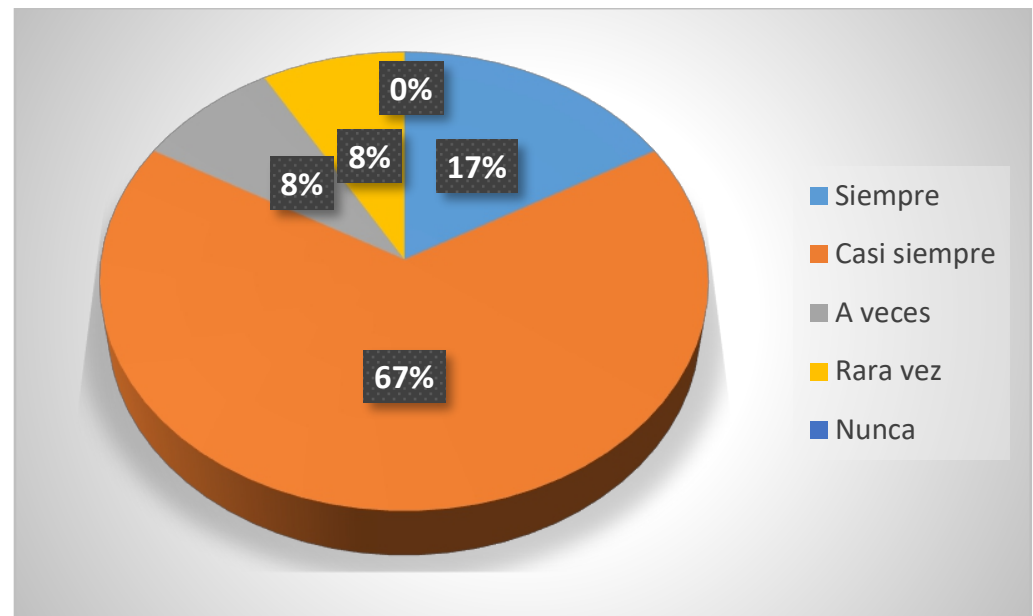

Gráfico 4. Conexión inalámbrica a internet en todos los espacios físicos, para el aprendizaje del inglés

\section{Análisis e Interpretación}

En el ítem 4, el 67\% de los docentes registró que Casi siempre la institución donde labora presta servicio de conexión inalámbrica a internet en todos los espacios físicos, para el aprendizaje del inglés, el 17\% Siempre, el $8 \%$ A veces; el $8 \%$ rara vez; mientras que no se registró frecuencias en la opción Siempre.

La gran mayoría de los docentes manifestó tener conexión inalámbrica a internet en todos los espacios físicos de la Unidad Educativa Pedro Vicente Maldonado. Un 17\% mencionó que siempre cuenta con conexión a internet, lo que significa no será problema el internet al momento de implementar el aprendizaje electrónico móvil (Podcast) para desarrollar la comprensión auditiva del inglés en los estudiantes.

Ítem 5: ¿Existe interactividad entre docente - estudiante, estudiantesestudiantes al momento de practicar una actividad de listening en un dispositivo móvil? 
Tabla 5. Interactividad entre docente - estudiante, estudiantes-estudiantes al momento de practicar una actividad de listening en un dispositivo móvil

\begin{tabular}{ccc}
\hline Escalas & Frecuencias & Porcentaje \\
\hline Siempre & 2 & $16 \%$ \\
Casi siempre & 2 & $17 \%$ \\
A veces & 2 & $17 \%$ \\
Rara vez & 6 & $50 \%$ \\
Nunca & 0 & $0 \%$ \\
TOTAL & 12 & $100 \%$ \\
\hline
\end{tabular}

Fuente. Encuesta

Elaborado por. Ruiz Renato, 2017

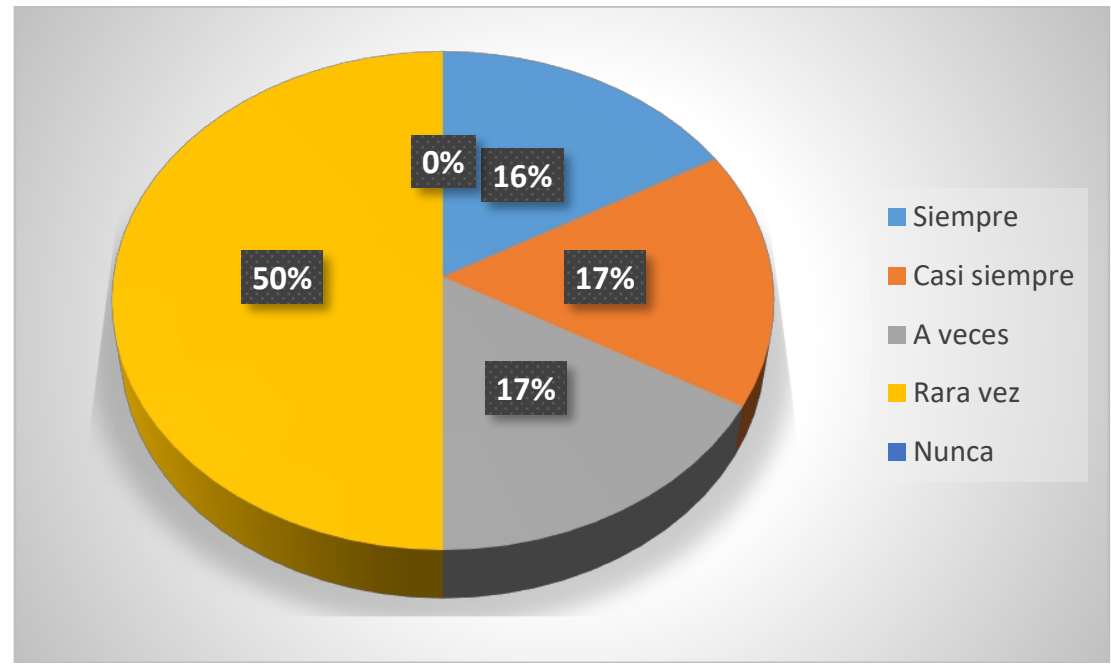

Gráfico 5. Interactividad entre docente - estudiante, estudiantes-estudiantes al momento de practicar una actividad de listening en un dispositivo móvil

\section{Análisis e Interpretación}

En el ítem 5, el 50\% de los docentes registró que rara vez existe interactividad entre docente - estudiante, estudiantes - estudiantes al momento de practicar una actividad de listening en un dispositivo móvil, el $17 \%$ A veces y Casi siempre, el 16\% Siempre; mientras que no se registró frecuencias en la opción Nunca.

Se pudo apreciar que la mitad de los encuestados manifiestan que rara vez existe interactividad entre docente - estudiante, estudiante - estudiante al momento de practicar una actividad de escucha en clase; lo que se traduce en una imperiosa necesidad de incrementar el porcentaje de interacción entre docentes y estudiantes, así como entre estudiantes.

El 17\% de docentes encuestados registró que a veces existe interacción al momento de practicar una actividad de escucha en clase; por lo tanto, con la implementación del aprendizaje electrónico móvil para desarrollar la comprensión auditiva del inglés, se podría incrementar el porcentaje de interacción existente. 
Ítem 6: ¿Sus estudiantes relacionan la gramática aprendida en clase con los diálogos escuchados en inglés?

Tabla 6. Sus estudiantes relacionan la gramática aprendida en clase con los diálogos escuchados en inglés?

\begin{tabular}{ccc}
\hline Escalas & Frecuencias & Porcentaje \\
\hline Siempre & 1 & $8 \%$ \\
Casi siempre & 2 & $17 \%$ \\
A veces & 4 & $34 \%$ \\
Rara vez & 4 & $33 \%$ \\
Nunca & 1 & $8 \%$ \\
TOTAL & 12 & $100 \%$ \\
\hline
\end{tabular}

Fuente. Encuesta

Elaborado por. Ruiz Renato, 2017

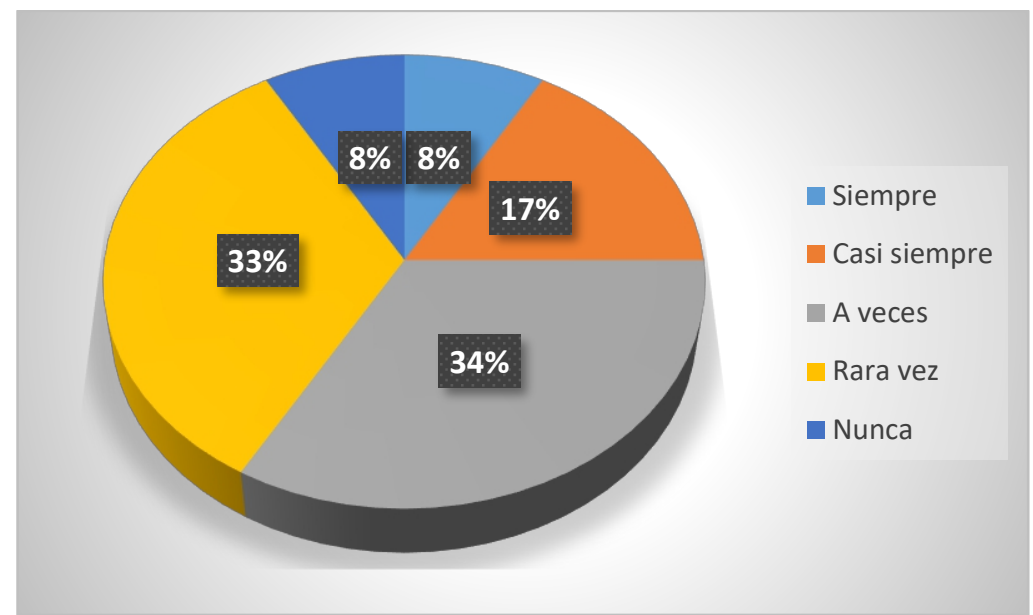

Gráfico 6. Sus estudiantes relacionan la gramática aprendida en clase con los diálogos escuchados en inglés?

\section{Análisis e Interpretación}

En el ítem 6, el $34 \%$ de los docentes registró que $A$ veces sus estudiantes relacionan la gramática aprendida en clase con los diálogos escuchados en inglés, el 33\% Rara vez, el 17\% Casi siempre; mientras que el 8\% siempre y nunca;

Apenas 4 docentes encuestados manifiestaron que sus estudiantes a veces relacionan la gramática aprendida en clase con los diálogos escuchados en inglés; mientras que otros 4 manifiesta que rara vez sus estudiantes relacionan lo que aprenden en clase, con lo que escuchan de un audio. Por este motivo, la presente investigación resulta de gran ayuda para que los estudiantes al desarrollar su nivel de compresión auditiva gracias al Podcast, puedan asociar la gramática aprendida con los diálogos que escuchan en la lista de reproducción del Podcast, o en cualquier otro archivo de audio, o conversación que escuchen. 
Ítem 7: ¿Sus estudiantes logran comprender los contenidos y mensajes compartidos en inglés fácilmente?

Tabla 7: Estudiantes logran comprender los contenidos y mensajes compartidos en inglés fácilmente

\begin{tabular}{ccc}
\hline Escalas & Frecuencias & Porcentaje \\
\hline Siempre & 0 & $0 \%$ \\
Casi siempre & 1 & $8 \%$ \\
A veces & 3 & $25 \%$ \\
Rara vez & 7 & $58 \%$ \\
Nunca & 1 & $8 \%$ \\
TOTAL & 12 & $100 \%$ \\
\hline
\end{tabular}

Fuente. Encuesta

Elaborado por. Ruiz Renato, 2017

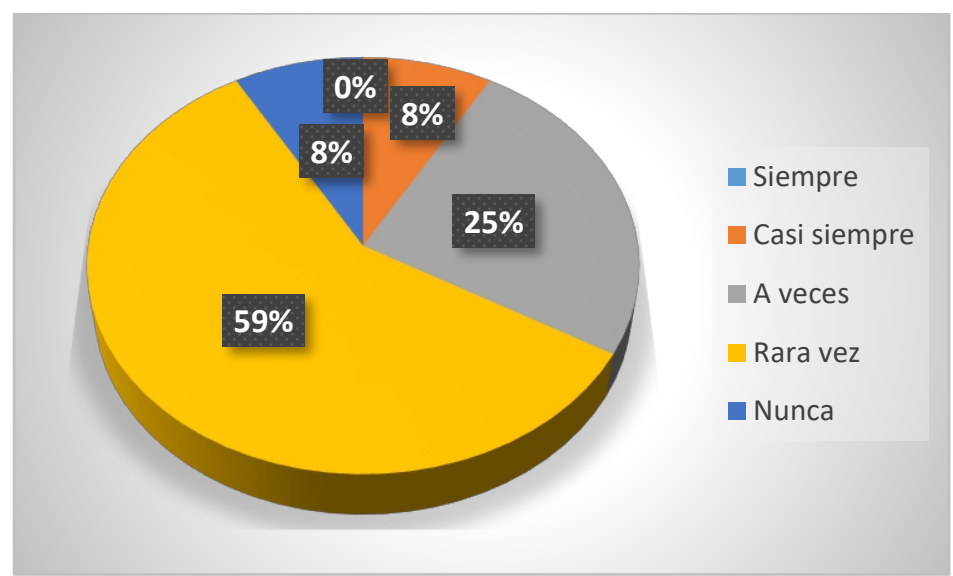

Gráfico 7. Estudiantes logran comprender los contenidos y mensajes compartidos en inglés fácilmente

\section{Análisis e Interpretación}

En el ítem 7, el 59\% de los docentes registró que Rara vez sus estudiantes logran comprender los contenidos y mensajes compartidos en inglés fácilmente, el $25 \%$ A veces, el $8 \%$ Casi siempre y nunca; mientras que no se registraron frecuencias en la opción Siempre.

Fue posible interpretar que la mayoría de encuestados manifestaron que sus estudiantes rara vez logran comprender los contenidos y mensajes compartidos en inglés, lo que quiere decir que los estudiantes tienen una grave falencia en el entendimiento del idioma a través de la escucha.

Ítem 8: ¿Cree usted que es importante desarrollar el nivel de comprensión auditiva de los estudiantes a través de recursos móviles? 
Tabla 8. Importancia del desarrollar del nivel de comprensión auditiva de los estudiantes a través de recursos móviles

\begin{tabular}{ccc}
\hline Escalas & Frecuencias & Porcentaje \\
\hline Siempre & 7 & $59 \%$ \\
Casi siempre & 3 & $25 \%$ \\
A veces & 1 & $8 \%$ \\
Rara vez & 1 & $8 \%$ \\
Nunca & 0 & $0 \%$ \\
TOTAL & 12 & $100 \%$ \\
\hline
\end{tabular}

Fuente. Encuesta

Elaborado por. Ruiz Renato, 2017

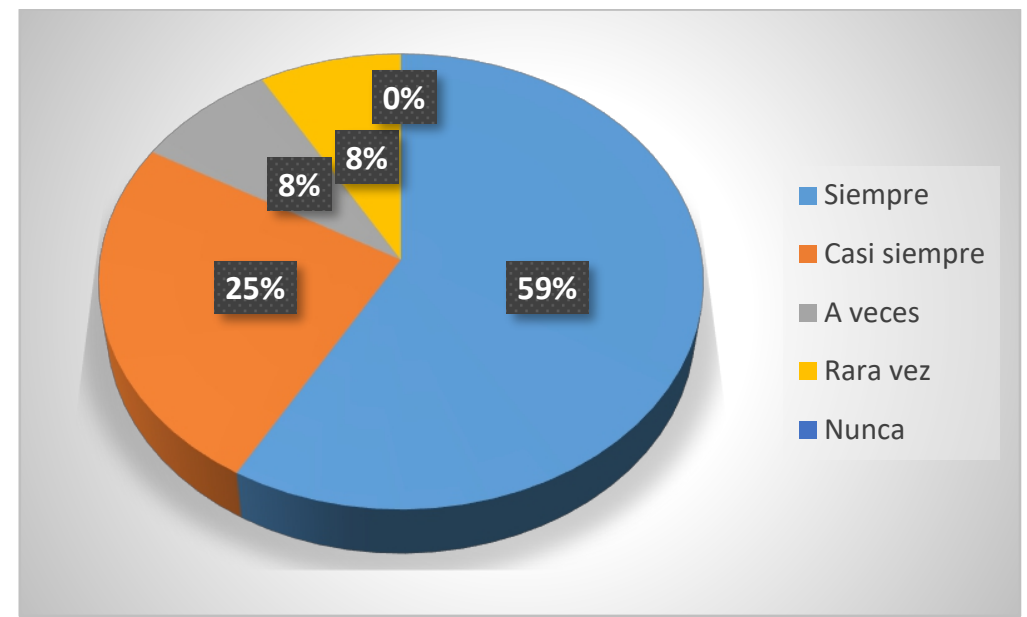

Gráfico 8. Importancia del desarrollar del nivel de comprensión auditiva de los estudiantes a través de recursos móviles

Análisis e Interpretación

En el ítem 8, el 59\% de los docentes registró que Siempre es importante desarrollar el nivel de comprensión auditiva de los estudiantes a través de recursos móviles, el $25 \%$ casi siempre, el $8 \%$ A veces y rara vez; mientras que no se registraron frecuencias en la opción Nunca.

Fue posible interpretar que la mayoría de encuestados registraron que es importante desarrollar el nivel de compresión auditiva de los estudiantes a través de recursos móviles.

Ítem 9: ¿Usted monitorea si sus estudiantes entendieron el mensaje de la actividad en inglés en las diferentes etapas (antes, durante y después)? 
Tabla 9: Usted monitorea si sus estudiantes entendieron el mensaje de la actividad en inglés en las diferentes etapas (antes, durante y después)

\begin{tabular}{ccc}
\hline Escalas & Frecuencias & Porcentaje \\
\hline Siempre & 5 & $42 \%$ \\
Casi siempre & 4 & $33 \%$ \\
A veces & 3 & $25 \%$ \\
Rara vez & 0 & $0 \%$ \\
Nunca & 0 & $0 \%$ \\
TOTAL & 12 & $100 \%$ \\
\hline
\end{tabular}

Fuente. Encuesta

Elaborado por. Ruiz Renato, 2017

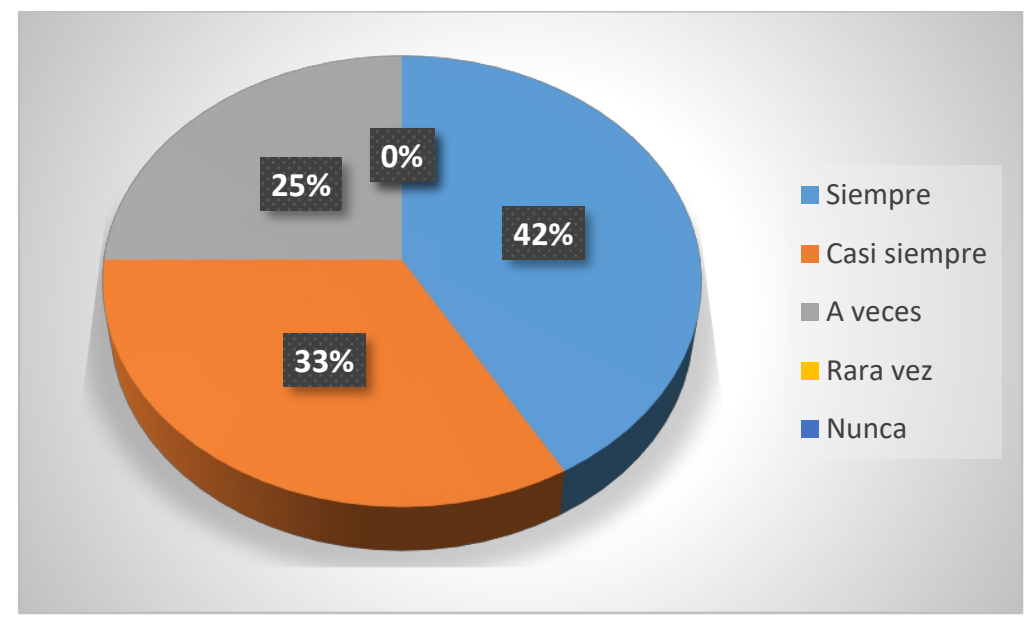

Gráfico 9. Usted monitorea si sus estudiantes entendieron el mensaje de la actividad en inglés en las diferentes etapas (antes, durante y después)

\section{Análisis e Interpretación}

En el ítem 9, el 42\% de los docentes registró que Siempre monitorea, si sus estudiantes entendieron el mensaje de la actividad en inglés en las diferentes etapas (antes, durante y después), el 33\% Casi siempre, el 25\% A veces; mientras que no se registraron frecuencias en la opción Rara vez y Nunca.

De acuerdo a las respuestas obtenidas previamente, se pudo registrar que el resultado del monitoreo realizado por los docentes, los estudiantes no comprenden el mensaje impartido en inglés luego de una actividad.

Ítem 10: ¿Envía usted a sus estudiantes tareas relacionadas con el uso de tecnologías móviles para el desarrollo de la compresión auditiva? 
Tabla 10. Envía usted a sus estudiantes tareas relacionadas con el uso de tecnologías móviles para el desarrollo de la compresión auditiva

\begin{tabular}{ccc}
\hline Escalas & Frecuencias & Porcentaje \\
\hline SIEMPRE & 1 & $8 \%$ \\
CASI SIEMPRE & 4 & $34 \%$ \\
AVECES & 5 & $42 \%$ \\
RARA VEZ & 1 & $8 \%$ \\
NUNCA & 1 & $8 \%$ \\
TOTAL & 12 & $100 \%$ \\
\hline
\end{tabular}

Fuente. Encuesta

Elaborado por. Ruiz Renato, 2017

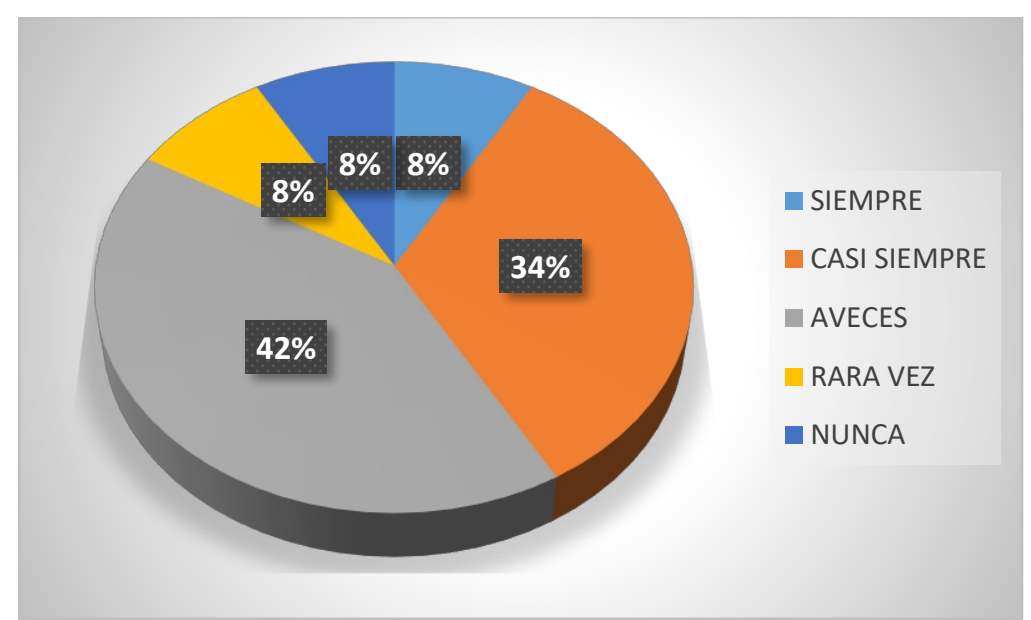

Gráfico 10: Envía usted a sus estudiantes tareas relacionadas con el uso de tecnologías móviles para el desarrollo de la compresión auditiva

\section{Análisis e Interpretación}

En el ítem 9, el 42\% de los docentes registró que $A$ veces envía a sus estudiantes tareas relacionadas con el uso de tecnologías móviles para el desarrollo de la compresión auditiva, el 34\% casi siempre; mientras que el 8\% registran las opciones Siempre, Rara vez y Nunca.

En el último ítem de la encuesta, es posible observar que 5 docentes manifiestan que a veces envían a sus estudiantes tareas relacionadas con el uso de tecnologías móviles, para el desarrollo de la compresión, lo cual permite que la presente investigación se desarrolle de mejor forma, ya que los estudiantes están familiarizados con el uso de tecnologías móviles para el desarrollo de sus tareas de inglés.

\section{Conclusion}

La identificación de los principios metodológicos que guían el uso de podcast como herramienta didáctica en la enseñanza del idioma inglés con 
énfasis en la destreza de la comprensión auditiva sirvió como instrumento para su aplicación cronológica y sistemática.

La evaluación obtenida mediante la encuesta permitió conocer cuantitativamente la aplicación del aprendizaje electrónico móvil aplicado por los docentes de la Unidad Educativa Pedro Vicente Maldonado; la misma que determinó su impacto positivo en la reutilización de los recursos a través de la tecnología, permitiendo superar las actividades tradicionales.

Es evidente que las actividades orientadas con los podcasts son importantes en el desarrollo de los procesos de enseñanza como nuevos paradigmas en la educación; pues impulsó y motivó a los docentes en a la utilización de las TIC como alternativas tecnológicas de soporte para transmitir el conocimiento del idioma inglés.

\section{References:}

1. Álvarez F. (2014). Universidad Nacional de Educación a Distancia de Madrid. Tesis Doctoral "El podcasting en la enseñanza de las lenguas extranjeras".

2. Baird, D. E. \& Fisher, M. (2006). NEO MILLENNIAL USER EXPERIENCE DESIGN STRATEGIES: UTILIZING SOCIAL NETWORKING MEDIA TO SUPPORT "ALWAYS ON" LEARNING STYLES, Journal of Educational Technology Systems, VOL. 34, Nº 1 ; pp. 5-32.

3. Boulos, M.; Maramba, I. \& Wheeler, S. (2006). WIKIS, BLOGS AND PODCASTS: A NEW GENERATION OF WEB-BASED TOOLS FOR VIRTUAL COLLABORATIVE CLINICAL PRACTICE AND EDUCATION, BMC Medical Education, VOL. 6, N. 41; pp.1-8

4. CDI, (2015). Universidad Nacional Autónoma de México. Artículo "La Web 2.0 y sus servicios como herramientas en el entorno educativo del siglo XXI"

5. Chan, A. \& Lee, M. (2005). An mp3 a day keeps the worries awayexploring the use of podcasting to address preconceptions and alleviate pre-class anxiety amon gst undergraduate information technology students. (Recuperado de http://www.csu.edu.au/division/studserv/sec/papers/chan.pdf)

6. Facultad de Ciencias de la Educación de la Universidad de Granada (2008/2009). Artículo "Podcasting as a teaching and learning tool in Higher Education". España.

7. Moura, A. \& Carvalho, A.A. (2006). PODCAST: POTENCIALIDADES NA EDUCAÇÃO, Prisma.Com, No 3; pp. 88-120.

8. Marcelo, J.F. y Martín, E. (2008). Podcasting. Guía Práctica. Madrid: Anaya Multimedia. Martín, L. (2009). Competencia estratégica para la 
comprensión auditiva en español como lengua extranjera. España. Gobierno de España. Ministerio de Educación.

9. Miller, M., \& Piller, M. (2005). Principal factors of an audio reading delivery mechanism - evaluating educational use of the iPod, Kommers, P. y Richards, G. (Eds.). Proceedings of World Conference on Educational Multimedia, Hypermedia and Telecommunications 2005. Chesapeake: AACE; pp. 260-267

10. Rocael Hernández y Miguel Morales (2009). Universidad de Galileo. Artículo "Dispositivos móviles en la Educación"

11. Sánchez Vera, M. Universidad de Murcia. "Objetos de Aprendizaje de la asignatura Diseño y Evaluación de Materiales Educativos". 